\title{
Quintic B-Spline Collocation for Solving Abel's Integral Equation
}

\author{
Zahra Mahmoodi* \\ The Department of Mathematics, Abhar Branch, Islamic Azad University, Abhar, Iran. \\ * Corresponding author. Tel.: +989122165532; email: z_mahmoodi_a@yahoo.com \\ Manuscript submitted May 8, 2015; accepted September 29, 2015. \\ doi: 10.17706/ijapm.2015.5.4.243-251
}

\begin{abstract}
A collocation method based on quintic B-spline is presented for the numerical solution of Abel's integral equation. The solution is collocated by quintic B-spline and then the integral equation is approximated by the product trapezoidal formula. The error analysis of proposed numerical scheme is studied theoretically. The presented method is tested with two examples and numerical results are given to illustrate the efficiency of the proposed method.
\end{abstract}

Key words: Abel's integral equation, quintic B-spline, product trapezoidal, error analysis.

\section{Introduction}

We consider the integral equation of the Abel type

$$
\int_{0}^{T}(t-x)^{-\mu} k(t, x) y(x) d x=f(t), \quad t \in[0, T]
$$

where $0<\mu<1$ and $k$ and $f$ are given functions and $y$ is unknown function. Equations of this type arise in the polymer physics, atmospheric physics and many other fields see [1]-[3], thus application of numerical methods for solving this equations are attractive. To solve the Abel's integral equation, several numerical approaches have been proposed, such as the discrete super convergence properties of spline collocation [4], [5], the iterative algorithms [6], the standard numerical integration rules with one or more starting values [7], the Adomian Decomposition and Product Integration Methods [8], the Homotopy analysis method [9], the fractional calculus [10]-[12], the Bernstein polynomials [13], two different numerical methods with comparison [14], the Superconvergent Nyström and degenerate kernel methods [15], the variational iteration method [16], the Analytical-Approximate solution [17], the Chebyshev polynomials [18], [19], the Haar wavelet method [20], the double integral transform [21], the exact multiplicity of positive solutions of the singular semilinear equation with Dirichlet boundary condition [22]. In this paper we propose a product integration to approximate the Volterra integral equation and quintic B-spline collocation to approximate the unknown function.

\section{Quintic B-Spline Collocation Method}

We introduce the quintic B-spline space and basis functions to construct an interpolant $s$ to be used in formulation of quintic B-spline collocation method. Let $\Delta_{n}:\left\{a=t_{0}<t_{1}<\cdots<t_{n}=b\right\}$ be a uniform partition 
of the interval $[a, b]$ with step size $h=\frac{b-a}{n}$. The quintic B-spline space denote by $S_{5}\left(\Delta_{n}\right)=\left\{s \in C^{4}[a, b] ;\left.s\right|_{\left[t_{i}, t_{i+1}\right]} \in \mathrm{P}_{5}, i=0,1, \cdots, n-1\right\}$, where $\mathrm{P}_{5}$ is the class of quintic polynomials. The construction of the quintic B-spline interpolate $s$ to the analytical solution $y$ for (1) can be performed with the help of the ten additional knots such that $t_{-5}<t_{-4}<t_{-3}<t_{-2}<t_{-1}$ and $t_{n+1}<t_{n+2}<t_{n+3}<t_{n+4}<t_{n+5}$.

Following [23], [24] we can define the B-spline functions

$$
\left\{\begin{array}{l}
B_{i}^{0}(t)= \begin{cases}1 \quad \text { if } t_{i} \leq t \leq t_{i+1} \\
0 \quad \text { otherwise }\end{cases} \\
B_{i}^{k}(t)=\left(\frac{t-t_{i}}{t_{i+k}-t_{i}}\right) B_{i}^{k-1}(t)+\left(\frac{t_{i+k+1}-t}{t_{i+k+1}-t_{i+1}}\right) B_{i+1}^{k-1}(t), \quad k \geq 1,
\end{array}\right.
$$

We consider a quintic B-spline $s(t)$ of the form

$$
s(t)=\sum_{i=-2}^{n+2} c_{i} B_{i}^{5}(t)
$$

where

$$
B_{i}^{5}(t)=\frac{1}{h^{5} 5 !}\left\{\begin{array}{lr}
\left(t-t_{i-3}\right)^{5}, & \text { if } t \in\left[t_{i-3}, t_{i-2}\right) \\
\left(t-t_{i-3}\right)^{5}-6\left(t-t_{i-2}\right)^{5}, & \text { if } t \in\left[t_{i-2}, t_{i-1}\right) \\
\left(t-t_{i-3}\right)^{5}-6\left(t-t_{i-2}\right)^{5}+15\left(t-t_{i-1}\right)^{5}, & \text { if } t \in\left[t_{i-1}, t_{i}\right) \\
\left(t-t_{i-3}\right)^{5}-6\left(t-t_{i-2}\right)^{5}+15\left(t-t_{i-1}\right)^{5}-20\left(t-t_{i}\right)^{5}, & \text { if } t \in\left[t_{i}, t_{i+1}\right) \\
\left(t-t_{i-3}\right)^{5}-6\left(t-t_{i-2}\right)^{5}+15\left(t-t_{i-1}\right)^{5}-20\left(t-t_{i}\right)^{5}+15\left(t-t_{i+1}\right)^{5}, & \text { if } t \in\left[t_{i+1}, t_{i+2}\right) \\
\left.\left(t-t_{i-3}\right)^{5}-6\left(t-t_{i-2}\right)^{5}+15\left(t-t_{i-1}\right)^{5}\right)-20\left(t-t_{i}\right)^{5}+15\left(t-t_{i+1}\right)^{5}-6\left(t-t_{i+2}\right)^{5}, & \text { if } t \in\left[t_{i+2}, t_{i+3}\right) \\
0 & t<t_{i-3}, t_{i+3}<t,
\end{array}\right.
$$

satisfying the following interpolatory conditions $s\left(t_{i}\right)=y\left(t_{i}\right), \quad 0 \leq i \leq n$, and the end conditions

$$
\left\{\begin{array}{l}
\left(\text { i) } D^{j} s\left(t_{0}\right)=D^{j} y\left(t_{0}\right), D^{j} s\left(t_{n}\right)=D^{j} y\left(t_{n}\right), j=1,2, \quad\right. \text { or } \\
(\text { ii }) D^{j} s\left(t_{0}\right)=D^{j} s\left(t_{n}\right), \quad j=1,2,3,4, \text { or } \\
\text { (iii) } D^{j} s\left(t_{0}\right)=0, \quad D^{j} s\left(t_{n}\right)=0, \quad j=3,4 .
\end{array}\right.
$$

\section{The Product Trapezoidal Method for Abel Equation}

The solution of equation (1) can be replaced with quintic B-spline equation we obtain:

$$
f(t)=\int_{0}^{T}(t-x)^{-\mu} k(t, x) s(x) d x, \quad t \in[0, T],
$$


We now collocate Eq.(6) at collocation points $t_{i}=i h, h=\frac{T}{n}, i=0, \ldots, n$ as

$$
f\left(t_{i}\right)=\int_{0}^{t_{i}}\left(t_{i}-x\right)^{-\mu} k\left(t_{i}, x\right) s(x) d x
$$

the next step is the product trapezoidal method which isconstructed by approximating $k(t, x) s(x)$ by piecewise linear functions [7], in particular

$$
k(t, x) s(x) \frac{x-t_{j}}{h} k\left(t, t_{j+1}\right) s\left(t_{j+1}\right)+\frac{t_{j+1}-x}{h} k\left(t, t_{j}\right) s\left(t_{j}\right), x \in\left[t_{j}, t_{j+1}\right],
$$

this leads to the integration formula

$$
\int_{0}^{t_{i}}\left(t_{i}-x\right)^{-\mu} k\left(t_{i}, x\right) s(x) d x w_{i, 0} k\left(t_{i}, t_{0}\right) s\left(t_{0}\right)+\sum_{j=1}^{i-1} w_{i, j} k\left(t_{i}, t_{j}\right) s\left(t_{j}\right)+w_{i, i} k\left(t_{i}, t_{i}\right) s\left(t_{i}\right),
$$

When

$$
\begin{aligned}
& w_{i, 0}=\frac{1}{h} \int_{t_{0}}^{t_{1}}\left(t_{1}-x\right)\left(t_{i}-x\right)^{\frac{-1}{2}} d x \\
& w_{i, j}=\frac{1}{h}\left(\int_{t_{j}}^{t_{j+1}}\left(t_{j+1}-x\right)\left(t_{i}-x\right)^{\frac{-1}{2}} d x+\int_{t_{j-1}}^{t_{j}}\left(x-t_{j-1}\right)\left(t_{i}-x\right)^{\frac{-1}{2}} d x\right), \\
& w_{i, i}=\frac{1}{h} \int_{t_{i-1}}^{t_{i}}\left(x-t_{i-1}\right)\left(t_{i}-x\right)^{\frac{-1}{2}} d x .
\end{aligned}
$$

To approximate the integral Eq.(7), we can use the product trapezoidal rule (9), we get the following $n \times(n+5)$ nonlinear system

$$
f\left(t_{i}\right)=w_{i, 0} k\left(t_{i}, t_{0}\right) s\left(t_{0}\right)+\sum_{j=1}^{i-1} w_{i, j} k\left(t_{i}, t_{j}\right) s\left(t_{j}\right)+w_{i, i} k\left(t_{i}, t_{i}\right) s\left(t_{i}\right), 1 \leq i \leq n .
$$

We impose the end conditions (5) but we can obtain a system of $(n+4) \times(n+5)$, this method requires a starting value $s(0)$. One possible way to get this is to consider (1) in the limit as $t$ approaches zero. Following [7], if $f(t)$ is continuous in $0 \leq t \leq t_{i}$

and

$$
\operatorname{Lim}_{t \rightarrow 0} t^{\alpha} f(t)=C
$$

where $C \neq 0$ and $\alpha<\mu$, then (1) has the solution

$$
s(0)= \begin{cases}0 & \text { if } \mu-\alpha>1, \\ \frac{c \Gamma(1-\alpha)}{\Gamma(1-\mu) k(0,0)} & \text { if } \mu-\alpha=1 .\end{cases}
$$


Hence by associate equations (5) and (11) with (13) we have the following linear system $(n+5) \times(n+5)$,

$$
\left\{\begin{array}{l}
s(0)-\frac{c \Gamma(1-\alpha)}{\Gamma(1-\mu) k(0,0)}=0, \quad \sum_{j=0}^{i} w_{i, j} k\left(t_{i}, t_{j}\right) s\left(t_{j}\right)-f\left(t_{i}\right)=0, \quad 1 \leq i \leq n, \\
D^{j} s\left(t_{0}\right)=0, \quad D^{j} s\left(t_{n}\right)=0, \quad j=3,4,
\end{array}\right.
$$

by solving the above linear system via iterative method we determine the coefficients $c_{i}, i=-2, \ldots, n+2$ by setting $c_{i}$ in (3), we obtain the approximate solution for Eq.(1).

\section{Error Analysiss}

We analyze convergence theorem for the Abel equation. To obtain the error estimation of our approximation first, we recall the following definitions and theorem in [7], [25], [26].

Definition1. Let $y$ be the solution of the Abel equation (1), approximated by the product trapezoidal method then the function

$$
E\left(h, t_{i}\right)=\int_{0}^{t_{i}} p\left(t_{i}, x\right) k\left(t_{i}, x\right) y(x) d x-\sum_{j=0}^{i} w_{i, j} k\left(t_{i}, t_{j}\right) y\left(t_{j}\right)
$$

(where $\left.p\left(t_{i}, x\right)=\left(t_{i}-x\right)^{-\mu}\right)$ is the local consistency error of the method.

Definition2. Let $F$ be a class of equations of the form (1). If for every equation in $F$,

$$
\operatorname{Lim}_{h \rightarrow 0} \max _{0 \leq i \leq n}\left(\left|E\left(h, t_{i}\right)\right|\right)=0
$$

then the approximation method (11) is said to be consistent with (1) for the class of equations $F$.

If for every equation in $F$, there exists a constant $m$ such that

$$
\max _{0 \leq i \leq n}\left(\left|E\left(h, t_{i}\right)\right|\right) \leq m h^{q},
$$

then the method is said to be consistent of order $q$ in $F$.

Theorem 1. Let $s(t)$ be the quintic B-spline interpolate for a given function $y \in C^{10}[a, b]$. Then for uniform partitions we have for $i=0, \ldots, n$,

$$
s_{i}^{\prime}=y_{i}^{\prime}+o\left(h^{6}\right), s_{i}^{\prime \prime}=y_{i}^{\prime \prime}+\frac{h^{4}}{720} y_{i}^{(6)}+o\left(h^{6}\right), s_{i}^{\prime \prime \prime}=y_{i}^{\prime \prime \prime}-\frac{h^{4}}{240} y_{i}^{(7)}+o\left(h^{6}\right), s_{i}^{\prime \prime \prime \prime}=y_{i}^{\prime \prime \prime}-\frac{h^{2}}{12} y_{i}^{(6)}+\frac{h^{4}}{240} y_{i}^{(8)}+o\left(h^{6}\right) .
$$

Furthermore the following interpolating error estimates hold

$$
\left\|(s-y)^{(j)}\right\|_{\infty}=o\left(h^{6-j}\right), \text { for } \quad j=0,1,2,3,4 .
$$

Proof. See [26]. 
Theorem 2. Assume that there exist constants $d_{1}, d_{2}, d_{3}$ and $q$, such that for all $h>0$,

$$
\begin{gathered}
\max _{1 \leq i \leq n}\left|k\left(t_{i}, t_{j}\right)-k\left(t_{i-1}, t_{j}\right)\right| \leq d_{1} h, \quad 0 \leq j \leq i-1, \\
\max _{1 \leq i \leq n}\left|E\left(h, t_{i}\right)-E\left(h, t_{i-1}\right)\right| \leq d_{2} h^{q}, \\
\max _{1 \leq i \leq n}\left|k\left(t_{i}, t_{i-\frac{1}{2}}\right)\right| \geq d_{3} .
\end{gathered}
$$

Proof. Condition (18) hold if $k(t, x)$ is differentiable with respect to $t$, while (20) follows from the assumption that $k(t, t)$ is bounded away from zero. To determine (19) see [7].

Theorem 3. The approximate method (11) applied to the Abel integral equation (1) is convergence if

$$
\left|e_{i}\right| \leq \frac{d_{1} h}{d_{3}} \sum_{j=0}^{i-1}\left|e_{j}\right|+\frac{d_{2} h^{q}}{w d_{3}}
$$

where $e_{j}=s_{j}-y_{j}, j=0, \ldots, i$ and $\max _{0 \leq i \leq n}\left|w_{i, j}\right|=W \neq 0$.

proof. Now we consider the convergence analysis of the Abel equation (1)

$$
\int_{0}^{t} p(t, x) k(t, x) y(x) d x=f(t), \quad t \in[0, T]
$$

We suppose that at $x=t=t_{i}=t_{0}+i h, i=0, \ldots, n, h=\frac{T}{n}$ the corresponding approximation method for Abel integral equation (22) is

$$
\sum_{j=0}^{i} w_{i, j} k\left(t_{i}, t_{j}\right) s\left(t_{j}\right)=f\left(t_{i}\right), \quad i=1, \ldots, n
$$

By discretizing (22) and approximate the integrand by the product trapezoidal rule, then we can obtain

$$
\int_{0}^{t_{i}} p\left(t_{i}, x\right) k\left(t_{i}, x\right) y(x) d x-f\left(t_{i}\right)=\sum_{j=0}^{i} w_{i, j} k\left(t_{i}, t_{j}\right) y\left(t_{j}\right)-f\left(t_{i}\right)+E\left(h, t_{i}\right)=0
$$

after simplification we have

$$
\sum_{j=0}^{i} w_{i, j} k\left(t_{i}, t_{j}\right) y\left(t_{j}\right)=f\left(t_{i}\right)-E\left(h, t_{i}\right)
$$

where 


$$
E\left(h, t_{i}\right)=\int_{0}^{t_{i}} p\left(t_{i}, x\right) k\left(t_{i}, x\right) y(x) d x-\sum_{j=0}^{i} w_{i, j} k\left(t_{i}, t_{j}\right) y\left(t_{j}\right) .
$$

Subtracting (25) from (23) we get

$$
\sum_{j=0}^{i} w_{i, j} k\left(t_{i}, t_{j}\right) e_{j}=E\left(h, t_{i}\right)
$$

where $e_{j}=s\left(t_{j}\right)-y\left(t_{j}\right), j=0, \ldots, i$. Now by replacing $i$ by $i-1$ in (26) we have

$$
\sum_{j=0}^{i-1} w_{i-1, j} k\left(t_{i-1}, t_{j}\right) e_{j}=E\left(h, t_{i-1}\right)
$$

Now subtracting (27) from (26) we get

$$
\sum_{j=0}^{i} w_{i, j} k\left(t_{i}, t_{j}\right) e_{j}-\sum_{j=0}^{i-1} w_{i-1, j} k\left(t_{i-1}, t_{j}\right) e_{j}=E\left(h, t_{i}\right)-E\left(h, t_{i-1}\right),
$$

then we get

$$
\begin{aligned}
& w_{i, i} k\left(t_{i}, t_{i}\right) e_{i}-\sum_{j=0}^{i-1}\left[w_{i, j} k\left(t_{i}, t_{j}\right)-w_{i-1, j} k\left(t_{i-1}, t_{j}\right)\right] e_{j}=E\left(h, t_{i}\right)-E\left(h, t_{i-1}\right), \\
& \Rightarrow\left|w_{i, i} k\left(t_{i}, t_{i}\right)\right|\left|e_{i}\right| \leq \sum_{j=0}^{i-1}\left|w_{i-1, j} k\left(t_{i-1}, t_{j}\right)-w_{i, j} k\left(t_{i}, t_{j}\right)\right|\left|e_{j}\right|+\left|E\left(h, t_{i-1}\right)-E\left(h, t_{i}\right)\right| .
\end{aligned}
$$

We know that $\max _{0 \leq i \leq n}\left|w_{i, j}\right|=W \neq 0$, for fixed $j$ we get

$$
W\left|k\left(t_{i}, t_{i}\right)\right|\left|e_{i}\right| \leq \sum_{j=0}^{i-1} W\left|k\left(t_{i-1}, t_{j}\right)-k\left(t_{i}, t_{j}\right)\right|\left|e_{j}\right|+\left|E\left(h, t_{i-1}\right)-E\left(h, t_{i}\right)\right|,
$$

by using Theorem (2) we get $W d_{3}\left|e_{i}\right| \leq W d_{1} h \sum_{j=0}^{i-1}\left|e_{j}\right|+d_{2} h^{q} \quad \Rightarrow\left|e_{i}\right| \leq \frac{d_{1} h}{d_{3}} \sum_{j=0}^{i-1}\left|e_{j}\right|+\frac{d_{2} h^{q}}{W d_{3}}$.

When $h \rightarrow 0$ then the above first and second terms are zero and also the first term in the above tends to zero because this term is due to interpolating of $y(t)$ by quintic B-spline (see Theorem(1)).

We get for a fixed $i,\left|e_{i}\right| \rightarrow 0$ as $h \rightarrow 0$.

\section{Numerical Examples}

We test our presented method on two examples to examine the applicability and efficiency of our method, all the programs run by Mathematica. 
Table 1. The Errors $\|E\|$ in Solution of Example 1 at Particular Points

\begin{tabular}{lllll}
\hline \hline $\mathrm{x}$ & $\mathrm{n}$ & 50 & 100 & 150 \\
\hline 0.1 & $3.67(-05)$ & $6.02(-06)$ & $1.53(-06)$ & $6.87(-07)^{*}$ \\
0.2 & $5.15(-05)$ & $8.40(-06)$ & $2.12(-06)$ & $9.49(-07)$ \\
0.3 & $5.58(-05)$ & $9.05(-06)$ & $2.28(-06)$ & $1.02(-06)$ \\
0.4 & $5.53(-05)$ & $8.93(-06)$ & $2.25(-06)$ & $1.00(-06)$ \\
0.5 & $5.26(-05)$ & $8.48(-06)$ & $2.13(-06)$ & $9.48(-07)$ \\
0.6 & $4.91(-05)$ & $7.91(-06)$ & $1.98(-06)$ & $8.82(-07)$ \\
0.7 & $4.55(-05)$ & $7.31(-06)$ & $1.83(-06)$ & $8.14(-07)$ \\
0.8 & $4.19(-05)$ & $6.73(-06)$ & $1.68(-06)$ & $7.49(-07)$ \\
0.9 & $3.86(-05)$ & $6.19(-06)$ & $1.55(-06)$ & $6.89(-07)$ \\
1 & $3.56(-05)$ & $5.70(-06)$ & $1.43(-06)$ & $6.34(-07)$ \\
\hline \hline
\end{tabular}

Table 2. The Errors $\|E\|$ in Solution of Example 2 at Particular Points

\begin{tabular}{|c|c|c|c|c|}
\hline $\mathrm{x}$ & 20 & 50 & 100 & 150 \\
\hline 0.1 & $3.12(-04)$ & $5.75(-05)$ & $1.45(-05)$ & $6.51(-06)$ \\
\hline 0.2 & $3.23(-04)$ & $5.33(-05)$ & $1.35(-05)$ & $6.03(-06)$ \\
\hline 0.3 & $3.04(-04)$ & $4.96(-05)$ & $1.26(-05)$ & $5.59(-06)$ \\
\hline 0.4 & $2.84(-04)$ & $4.63(-05)$ & $1.17(-05)$ & $5.20(-06)$ \\
\hline 0.5 & $2.67(-04)$ & $4.33(-05)$ & $1.09(-05)$ & $4.86(-06)$ \\
\hline 0.6 & $2.51(-04)$ & $4.07(-05)$ & $1.02(-05)$ & $4.57(-06)$ \\
\hline 0.7 & $2.37(-04)$ & $3.84(-05)$ & $9.65(-05)$ & $4.30(-06)$ \\
\hline 0.8 & $2.24(-04)$ & $3.63(-05)$ & $9.13(-05)$ & $4.07(-06)$ \\
\hline 0.9 & $2.13(-04)$ & $3.44(-05)$ & $8.65(-05)$ & $3.86(-06)$ \\
\hline 1 & $2.02(-04)$ & $3.27(-05)$ & $8.23(-05)$ & $3.66(-06)$ \\
\hline
\end{tabular}

Example 1. Consider the following Abel equation with exact solution $y(t)=\frac{1}{1+t}$,

$$
\frac{2 t}{\sqrt{t+1}} \tanh ^{-1}\left(\sqrt{\frac{t}{t+1}}\right)+2 \sqrt{t}=\int_{0}^{t} \frac{(1+t+x) y(x)}{\sqrt{t-x}} d x, t \in[0,1]
$$

We must obtain value $s(0)$ of (13) when $\frac{-1}{2}=\alpha<\mu=\frac{1}{2}$ as

$$
\lim _{t \rightarrow 0} t^{\frac{-1}{2}}\left(\frac{2 t}{\sqrt{t+1}} \tanh ^{-1}\left(\sqrt{\frac{t}{t+1}}\right)+2 \sqrt{t}\right)=2 \quad \Rightarrow \mu-\alpha=1 \Rightarrow s(0)=\frac{2 \Gamma(1.5)}{\Gamma(0.5)}=1 .
$$

Example 2. Consider the following Abel equation with exact solution $y(t)=t$,

$$
\frac{16}{15} t^{\frac{5}{2}}+\frac{4}{3} t^{\frac{3}{2}}=\int_{0}^{t} \frac{(1+x) y(x)}{\sqrt{t-x}} d x, \quad t \in[0,1]
$$

We must obtain value $s(0)$ of (13) when $-1.5=\alpha<\mu=\frac{1}{2}$ as

$$
\lim _{t \rightarrow 0} t^{\frac{-3}{2}}\left(\frac{16}{15} t^{\frac{5}{2}}+\frac{4}{3} t^{\frac{3}{2}}\right)=1.33333 \Rightarrow \mu-\alpha>1 \Rightarrow s(0)=0
$$


Now we apply the system of (14) and we solve these examples with $n=20,50,100,150$ the absolute errors in particular points are tabulated in Table 1 and Table 2.

\section{Conclusion}

This paper presents method to find the solution of the Abel's integral equation by using the product trapezoidal method and collocation quintic B-spline. The equation is converted to a system of linear algebraic equation in terms of the linear combination coefficients appearing in the representation of the solution in spline basic function. We verified that the presented method can be applied with large number of $n$. The presented method is stable because when $n$ is increasing the error in the solution is also decreasing.

\section{References}

[1] Matar, M. M. (2011). Boundary value problem for some fractional integro differential equations with nonlocal conditions. Int. J. Nonlin Sci, 11(1), 3-9.

[2] Adomian, G. (1986). Nonlinear Stochastic Operator Equations. Orlando, Florida: Academic Press.

[3] Adomian, G. (1994). Solving Frontier Problems of Physics: The Decomposition Method. Kluwer, Boston.

[4] Diogo, T., \& Lima, P. (2007). Collocation solutions of a weakly singular volterra integral equation. TEMA Tend Mat Appl. Comput, 8, pp. 229-238.

[5] Liang, H., \& Brunner, H. (2012). Discrete superconvergence of collocation solutions for first-kind volterra integral equations. Journal of Integral Equations and Applications, 24(3), 359-391.

[6] Abbasbandy, S., \& Babolian, E. (1995). Autmatic augmented galerkin algorithms for linear first kind integral equations: non-singular and weak-singular kernels. Bulletin Iran Math Soci, 21, 35-62.

[7] Linz, P. (1985). Analytical and numerical methods for Volterra equations. SIAM Philadelphia.

[8] Jazbi, B., Djalalvand, M., \& Garshaasbi, M. (2011). A Numerical algorithm based on Adomian decomposition and product integration methods to solve a class of nonlinear weakly singular integral equations. Int. J. Nonlin. Sci., 11(3), 353-357.

[9] Afroozi, G. A., Vahidi, J., \& Saeidy, M. (2010). Solving a class of two-dimensional linear and nonlinear Volterra integral equations by means of the homotopy analysis method. Int J NonlinSci., 9(2), 213-219.

[10] Avazzadeh, Z., Shafiee, B., \& Loghmani, G. B. (2011). Fractional calculus for solving abel's integral equations using chebyshev polynomials. Appl Math Sci., 5(45), 2207-2216.

[11] Mandal, B. N., \& Chakrabarti, A. (2011). Applied Singular Integral Equations. Taylor and Francis.

[12] Diethelm, K., \& Ford, N. J. (2012). Volterra integral equations and fractional calculus: Do neighboring solutions intersect. J Int. Equ. Appl., 24(1), 25-37.

[13] Alipour, M., \& Rostamy, D. (2011). Bernstein polynomials for solving Abel's integral equation. J Math ComputSci., 3(4), 403-412.

[14] Ali, B. M. (2012). Two numerical methods for Abel's integral equation with comparison. ARIMA $j, 15$, $1-10$.

[15] Allouch, C., Sablonnière, P., Sbibih, D., \& Tahrichi, M. (2012). Superconvergent nyström and degenerate kernel methods for integral equations of the second kind. J Int Equ. Appl., 24(4), 463-485.

[16] Prajapati, R. N., Mohan, R., \& Kumar, P. (2012). Numerical solution of generalized abel's integral equation by variational iteration method. American Journal of Computational Mathematics, 2, 312-315.

[17] Yaghobifar, M., Nik, L. N. M. A., \& Eshkuvatov, Z. K. (2011). Analytical-approximate solution of abel integral equations. Int. Math. Forum, 6(5), 211-221.

[18] Abdulkawi, M., Eshkuvatov, Z. K., Nik, L. N. M. A. (2009). On the semi-bounded solution of cauchy type singular integral equations of the first Kind. Eur J Pure Appl Math, 2(3), 462-472. 
[19]] Khader, M. M. (2012). Introducing an efficient modification of the homotopy perturbation method by using chebyshev polynomials. Arab J. Math. Sci., 18(1), 61-71.

[20] Gelik, I. (2012). Haar wavelet method for solving generalized Burgers-Huxley equation. Arab J Math Sci., 18(1), 25-37.

[21] Kili, M. A., \& Eltayeb, H. (2010). On the partial differential equations with non-constant coefficients and convolution method. Eur J Pure Appl Math, 3(1), 45-50.

[22] Peter, L. S. (2010). Exact multiplicity of positive solutions for a class of singular semilinear equations, differential equations and dynamical systems. 18(4), 415-429.

[23] Prenter, P. M. (1975). Spline and Variational Methods. New-York: John Wiley and Sons.

[24] Kincaid, D., \& Cheney, W. (1990). Numerical Analysis: Mathematics of Scientific Computing. The University of Texas at Austin.

[25] Delves, L. M., \& Mohamad, J. L. (1985). Computational Methods for Integral Equations. Cambridge University.

[26] Irodotou-Ellina, M., \& Houstis, E. N. (1988). An $o\left(h^{6}\right)$ Quintic spline collocation method for fourth order two-point boundary value problems. BIT 28, 288-301.

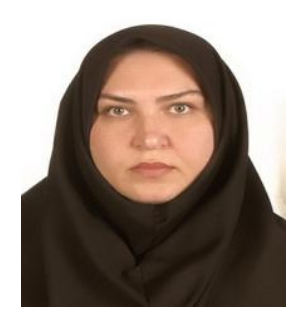

Zahra Mahmoodi received her Ph.D degree on mathematics from the Department of Mathematics of Islamic Azad University, Tehran, Iran 2012. Presently, she is the faculty member of the Islamic Azad University Mathematics Department and she is researching on numerical analysis, spline approximation and numerical method based on quintic B-spline has been developed to solve the nonlinear Fredholm and Volterra integral equations. 